\title{
Enhanced transmission with a graphene-dielectric microstructure at low-terahertz frequencies
}

\author{
Chandra S. R. Kaipa, ${ }^{1}$ Alexander B. Yakovlev, ${ }^{1, *}$ George W. Hanson, ${ }^{2}$ Yashwanth R. Padooru, ${ }^{1}$ \\ Francisco Medina, ${ }^{3}$ and Francisco Mesa ${ }^{4}$ \\ ${ }^{1}$ Department of Electrical Engineering, The University of Mississippi, University, Mississippi 38677, USA \\ ${ }^{2}$ Department of Electrical Engineering, University of Wisconsin-Milwaukee, Milwaukee, Wisconsin 53211, USA \\ ${ }^{3}$ Department of Electronics and Electromagnetism, University of Seville, Seville 41012, Spain \\ ${ }^{4}$ Department of Applied Physics 1, University of Seville, Seville 41012, Spain \\ (Received 28 March 2012; revised manuscript received 2 May 2012; published 5 June 2012)
}

\begin{abstract}
Here, we report on the transmissivity of electromagnetic waves through a stack of monolayer graphene sheets separated by dielectric slabs at low-terahertz frequencies. It is observed that the multilayer structure possesses band-gap properties and supports a series of bandpass and band-stop regions, similar to the cases of stacked metallic meshes separated by dielectric slabs at microwave/THz frequencies and a metal-dielectric stack at optical frequencies. The transmission resonances in the bandpass region are identified as coupled Fabry-Pérot resonances associated with the individual cavities of dielectric slabs loaded with graphene sheets. It is also noticed that these resonances lie within a certain characteristic frequency band, independent of the number of layers in the graphene-dielectric stack. The study is carried out using a simple analytical transfer-matrix approach or, equivalently, a circuit-theory model, resulting in the exact solution for the multiple dielectric/graphene sheet surface-conductivity model. Also, an independent verification of the observed phenomena is obtained with commercial numerical simulations.
\end{abstract}

DOI: 10.1103/PhysRevB.85.245407

PACS number(s): 78.20.-e, 41.20.Jb, 42.25.Bs, 42.70.Qs

\section{INTRODUCTION}

Electromagnetic wave interaction with periodic structures has been a subject of research for several decades. Of particular interest is high optical transmission through a thin-metaldielectric stack, ${ }^{1,2}$ in spite of extremely weak transmission through an individual isolated thin metal layer. The spectra for such a multilayer structure consist of a series of bandpass and band-stop regions. However, mimicking these properties in the microwave and far-infrared regimes is quite difficult due to the quasiperfect conductor behavior of metals at microwave and low-terahertz frequencies. To overcome this problem, it has recently been proposed ${ }^{3}$ to replace the thin metallic sheets of the optical system with metallic mesh grids (periodic arrays of perpendicularly crossed thin strips), wherein the transmission spectrum at microwaves includes several passband regions of high transmissivity associated with coupled Fabry-Pérot cavity resonances of the individual reactively loaded dielectric slabs. It should be noted that similar to the thin metal behavior at optical frequencies, a free-standing metallic mesh grid represents a partially reflective surface (PRS) with low transmissivity at microwave/THz frequencies. These observations are key points in the study presented in this paper.

In this work, we replace the wire mesh grids with graphene sheets, achieving a similar PRS functionality. In addition to this aspect, graphene is particularly interesting due to its unique thermal, mechanical, and electrical properties, which make it very useful in various electronic and electromagnetic applications. ${ }^{4,5}$ With the recent developments in the fabrication of graphene with large lateral dimensions, ${ }^{6,7}$ there have been numerous graphene applications at optical, infrared, and terahertz frequencies as tunable waveguiding interconnects, ${ }^{8}$ pn junctions, ${ }^{9}$ and waveguiding structures, ${ }^{10-12}$ among others. Recently, it has been shown that a surface plasmon mode can be strongly excited along a graphene monolayer with a point source, ${ }^{13,14}$ and experimental investigations have been performed for graphene-based plasmonic waveguides. ${ }^{15}$ In particular, the low-terahertz band has been of interest, with graphene used for frequency multiplication, ${ }^{16}$ plasmon oscillators, ${ }^{17}$ and cloaking. ${ }^{18}$ In this paper, we report on the transmissivity of electromagnetic waves through a stack of monolayer graphene sheets separated by dielectric slabs (with the geometry shown in Fig. 1). It is observed that, at low-terahertz frequencies (several $\mathrm{THz}$ ), resonances of high transmission occur, with the number of transmission peaks corresponding to the number of dielectric layers. These transmission resonances lie within a characteristic frequency band, independent of the number of layers, which correspond to the passband regime of an infinite periodic structure. A similar behavior has been observed with a stack of metallic meshes separated by dielectric slabs at microwaves ${ }^{3}$ (and, in general, $\mathrm{THz}$ frequencies) and with a thin-metal-dielectric stack at optical frequencies. ${ }^{2}$ However, graphene sheets used in the stack shown in Fig. 1 are atomically thin monolayers that behave as reactive inductive surfaces (with low real part and negative imaginary part of the surface conductivity of graphene at low-terahertz frequencies ${ }^{14,19}$ ). In addition, a graphene sheet has a low transmissivity at low-terahertz frequencies and behaves similar to that of a partially reflective surface at microwave/ $\mathrm{THz}$ frequencies. In this regard, a graphene monolayer at low-terahertz frequencies mimics the properties of a reactive inductive surface at microwave/ $\mathrm{THz}$ frequencies (for example, metallic mesh grid) as well as that of a thin solid metallic surface at optical frequencies.

\section{THEORY}

In the analysis to follow, graphene is characterized by the following surface conductivity $\sigma\left(\omega, \mu_{c}, \Gamma, T\right)$ model based on 


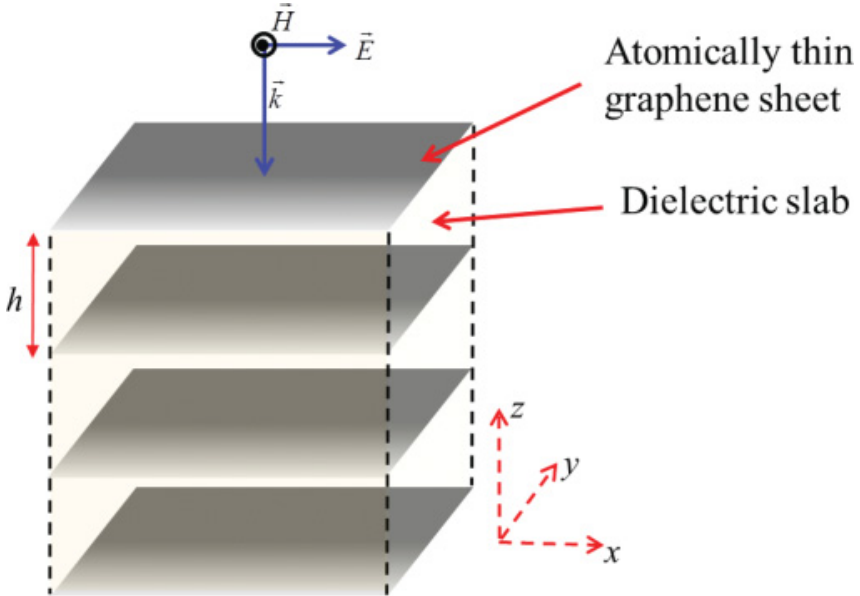

FIG. 1. (Color online) Geometry of a stack of atomically thin graphene sheets separated by dielectric slabs with a plane wave at normal incidence.

the Kubo formula ${ }^{19,20}$

$$
\begin{aligned}
\sigma\left(\omega, \mu_{c}, \Gamma, T\right)= & \frac{j e^{2}(\omega-j \Gamma)}{\pi \hbar^{2}} \\
& \times\left[\frac{1}{(\omega-j \Gamma)^{2}} \int_{0}^{\infty}\left(\frac{\partial f_{d}(\varepsilon)}{\partial \varepsilon}-\frac{\partial f_{d}(-\varepsilon)}{\partial \varepsilon}\right) \varepsilon d \varepsilon\right. \\
& \left.-\int_{0}^{\infty} \frac{f_{d}(-\varepsilon)-f_{d}(\varepsilon)}{(\omega-j \Gamma)^{2}-4(\varepsilon / \hbar)^{2}} d \varepsilon\right]
\end{aligned}
$$

where $-e$ is the charge of an electron, $\omega$ is the radian frequency, $\hbar=h / 2 \pi$ is the reduced Planck's constant, $f_{d}(\varepsilon)=$ $\left(e^{\left(\varepsilon-\mu_{c}\right) / k_{B} T}+1\right)^{-1}$ is the Fermi-Dirac distribution, $k_{B}$ is Boltzmann's constant, $T$ is temperature, $\varepsilon$ is the energy, $\mu_{c}$ is the chemical potential, and $\Gamma$ is the phenomenological scattering rate, which is assumed to be independent of energy $\varepsilon$. The first term in Eq. (1) is due to intraband contributions,

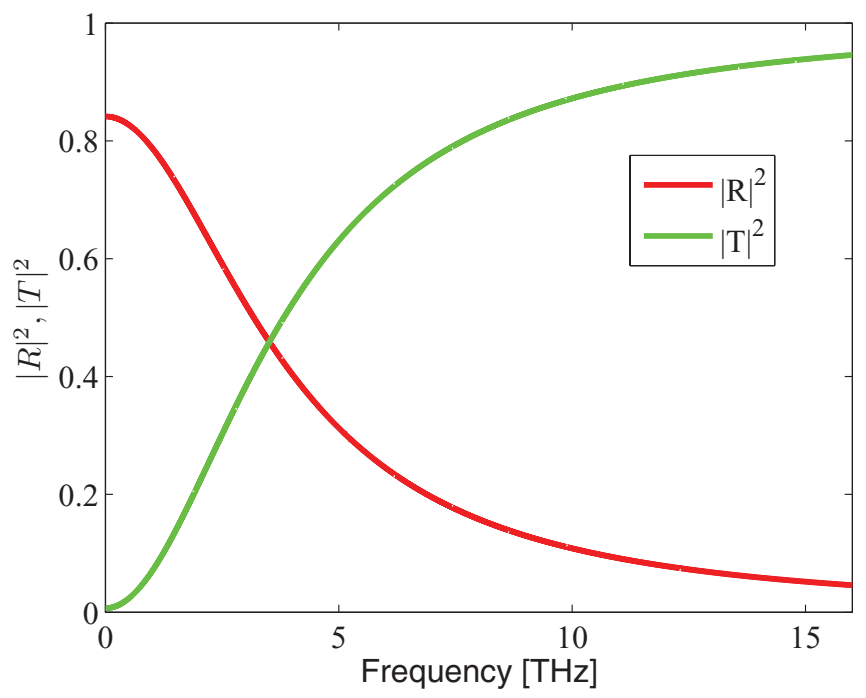

FIG. 2. (Color online) Reflectivity $|R|^{2}$ and transmissivity $|T|^{2}$ of a free-standing graphene sheet for $\mu_{c}=1 \mathrm{eV}$.

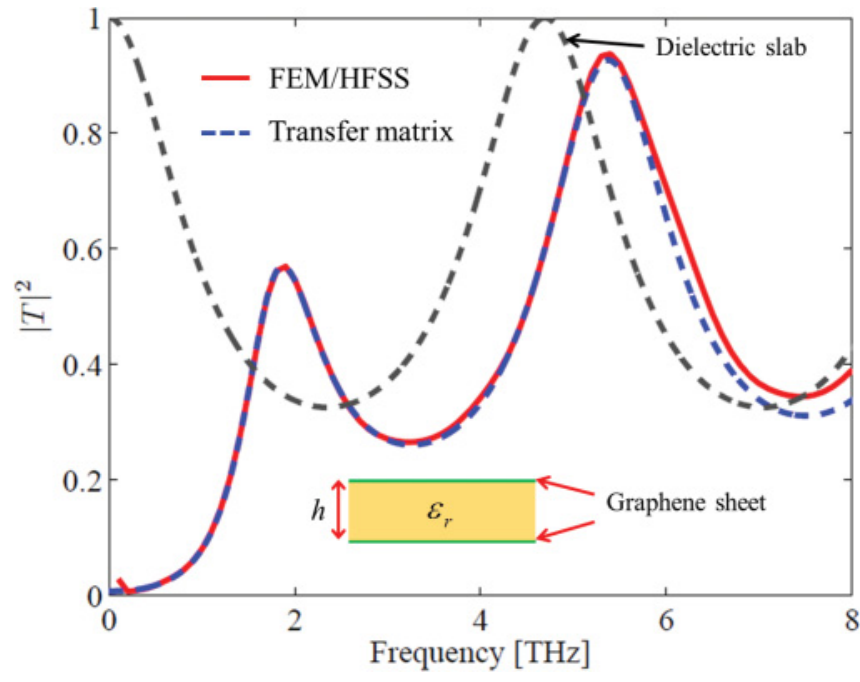

FIG. 3. (Color online) Transfer-matrix and FEM/HFSS results of the transmissivity $|T|^{2}$ for a two-sided graphene structure with a plane wave at normal incidence. Structural parameters: $h=10 \mu \mathrm{m}$, $\varepsilon_{r}=10.2$, and $\mu_{c}=0.5 \mathrm{eV}$.

and can be evaluated in closed form as ${ }^{19}$

$$
\sigma_{\text {intra }}=-j \frac{e^{2} k_{B} T}{\pi \hbar^{2}(\omega-j \Gamma)}\left[\frac{\mu_{c}}{k_{B} T}+2 \ln \left(e^{-\mu_{c} / k_{B} T}+1\right)\right] .
$$

The second term in Eq. (1) is due to interband contributions, and can be approximated for $k_{B} T \ll\left|\mu_{c}\right|, \hbar \omega$ as ${ }^{19}$

$$
\sigma_{\text {inter }}=\frac{-j e^{2}}{4 \pi \hbar} \ln \left(\frac{2\left|\mu_{c}\right|-(\omega-j \Gamma) \hbar}{2\left|\mu_{c}\right|+(\omega-j \Gamma) \hbar}\right) .
$$

From the above two expressions, it is found that, in the far-infrared regime, the contribution due to the interband electron transition is negligible below $10-15 \mathrm{THz}$, and needs to be taken into account at higher frequencies depending on the value of the chemical potential. ${ }^{19}$ Thus, the surface

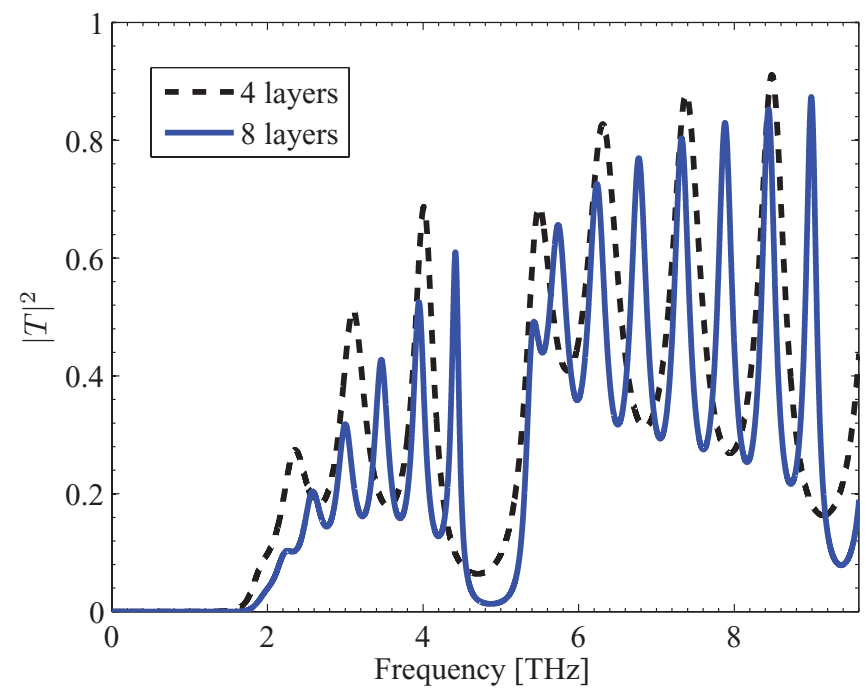

FIG. 4. (Color online) Transmissivity $|T|^{2}$ of four- and eightlayer graphene-dielectric stack structures. Structural parameters: $h=$ $10 \mu \mathrm{m}, \varepsilon_{r}=10.2$, and $\mu_{c}=1 \mathrm{eV}$. 

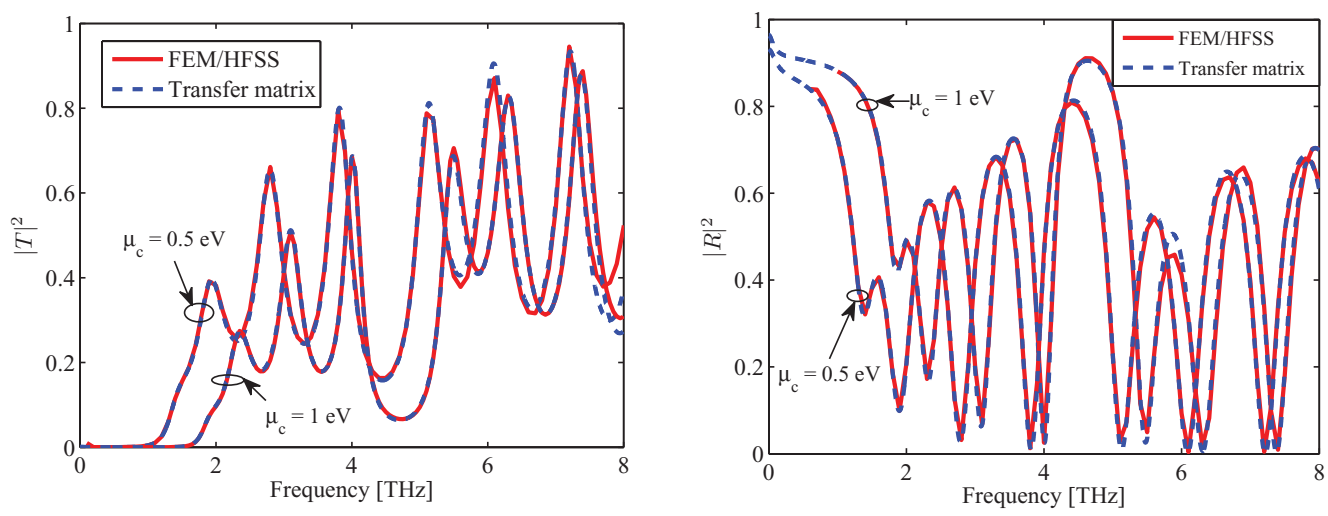

FIG. 5. (Color online) Transfer-matrix and FEM/HFSS results of the (a) transmissivity $|T|^{2}$ and (b) reflectivity $|R|^{2}$ for a four-layer graphene-dielectric stack with $\mu_{c}=0.5$ and $1 \mathrm{eV}$. Structural parameters: $h=10 \mu \mathrm{m}$ and $\varepsilon_{r}=10.2$.

conductivity of graphene is found to depend predominantly on intraband transitions [given by Eq. (2)], and is complex valued with a negative imaginary part (nevertheless, computations were made using both intraband and interband terms). This conductivity corresponds to the surface impedance of a graphene monolayer $Z_{s}=1 / \sigma$, which at low-terahertz frequencies behaves as a low-loss inductive surface due to small values of $\operatorname{Re}\{\sigma\}$. This behavior of the surface impedance is similar to that of the subwavelength metallic mesh grid at microwave/THz frequencies. ${ }^{3}$

With the graphene sheet characterized by a complex surface conductivity, and since the interaction in a graphene dielectric stack is by plane-wave reflection and transmission (no higher-order modes are excited), the transmissivity $|T|^{2}$ and the reflectivity $|R|^{2}$ of the graphene-dielectric stack can be obtained by applying the two-sided impedance boundary conditions at the graphene-dielectric interfaces ${ }^{19}$ with the use of a transfer-matrix approach for dielectric layers, resulting in the exact solution for the multiple dielectric/graphene sheet surface-conductivity model. Alternatively, the analysis can be carried out using the simple circuit-theory model described in Ref. 3, wherein graphene sheets are modeled as shunt admittances $Y_{s}=\sigma$. In what follows, the results obtained with this approach will be called transfer matrix. Also, an independent verification is obtained with a finite-element method (FEM) commercial simulation code [HFSS (Ref. 22)]. In this analysis, it is assumed that the lateral dimensions of the graphene are greater than a few tens of micrometer (i.e., much greater than the mean-free path of electrons).

\section{RESULTS AND DISCUSSIONS}

In all the simulations (transfer matrix and FEM/HFSS), $\Gamma=1 / \tau=1.32 \mathrm{meV}$ ( $\tau=0.5 \mathrm{ps}$, which corresponds to a mean-free path of several hundred nanometers), and $T=$ $300 \mathrm{~K}$. First, we consider the reflection and transmission properties of a free-standing graphene sheet in air. The results for the reflectivity $|R|^{2}$ and transmissivity $|T|^{2}$ shown in Fig. 2 are obtained as the solution for a plane-wave incidence with the sheet impedance boundary condition at the graphene interface with the surface impedance $Z_{s}=1 / \sigma$ [see also Eq. (35) in Ref. 19 for the reflection and transmission coefficients]. It can be seen that at low-terahertz frequencies (several
$\mathrm{THz}$ ), the transmissivity is low (reflectivity is high), and the graphene sheet behaves similar to an inductive PRS at microwave/ $\mathrm{THz}$ frequencies (for example, metallic mesh grid with sub-wavelength dimensions acting as a high-pass filter ${ }^{21}$ ).

Next, we consider the case of a dielectric layer (with thickness $h=10 \mu \mathrm{m}$ and permittivity $\varepsilon_{r}=10.2$ ) sandwiched between two graphene sheets (two-sided graphene structure). The transfer-matrix results of the transmissivity are depicted in Fig. 3, along with the simulation results obtained with commercial program HFSS (Ref. 22) (based on the finite element method). It can be seen that a transmission resonance appears at low frequencies [when compared to the typical Fabry-Pérot (FP) resonance of the dielectric slab without the graphene sheets], and is associated with the FP-type resonance of the dielectric slab loaded with graphene sheets. The graphene sheets play the role of reactive (inductive)

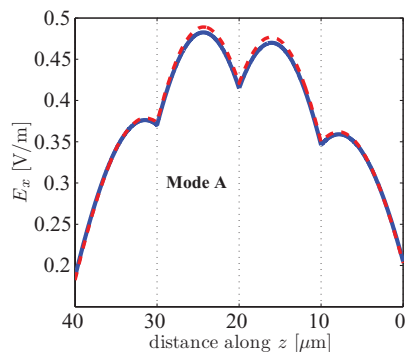

(a)

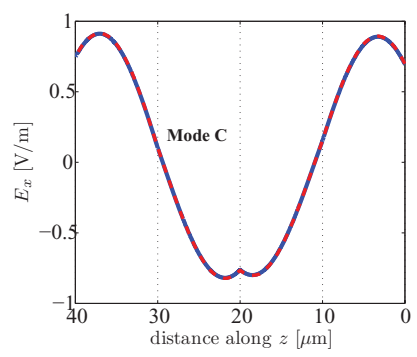

(c)

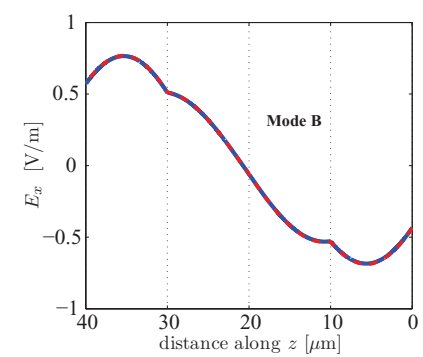

(b)

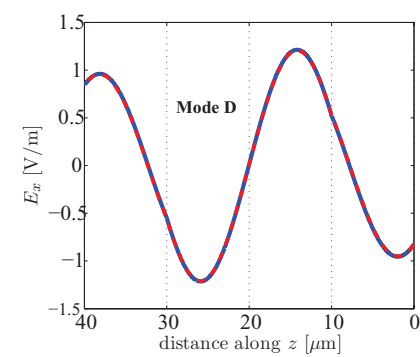

(d)
FIG. 6. (Color online) Electric-field distributions for the four resonance modes of the four open and coupled Fabry-Pérot cavities associated with the individual dielectric slabs in the stacked graphenedielectric structure. The FEM/HFSS (red curves) and transfer-matrix (blue curves) results show a very good agreement. 

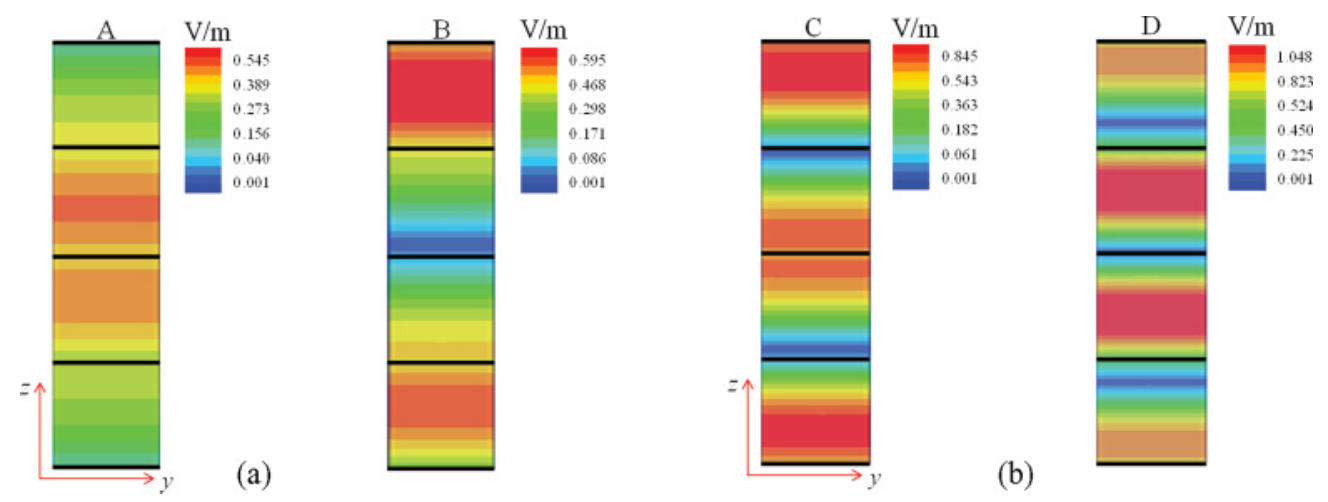

FIG. 7. (Color online) Magnitude of the total electric-field distributions of the four resonance modes in the four-layer graphene-dielectric stack calculated using HFSS.

loadings, which effectively increase the electrical length of the two-sided graphene-dielectric cavity. A similar effect can be observed with the mesh grid structure, ${ }^{3}$ wherein the inductive reactance of sub-wavelength grids corresponds to the stored magnetic energy of evanescent higher-order Floquet harmonics of a periodic structure (operating at frequencies below the diffraction limit). However, an advantage of the graphene sheets is that higher-order Floquet harmonics are not excited, and the inductive reactance of graphene is directly related to the properties of the material (such that at lowterahertz frequencies, $\operatorname{Im}\{\sigma\}<0$ and $\operatorname{Re}\{\sigma\}$ has relatively small values).

With a further increase in the number of identical layers (dielectric slabs with the same permittivity and graphene sheets biased with the same chemical potential), each single peak of the single-layer case is replaced by $N$ peaks of the $N$-layer case ( $N$ dielectric slabs and $N+1$ graphene sheets), as occurs in, e.g., atomic level splitting in forming molecules. Also, all these peaks lie in a characteristic frequency band (within a bandpass region followed by a band-stop region). The calculations based on the transfer-matrix method for the transmissivity of four- and eight-layer graphene structure are depicted in Fig. 4, showing the observed phenomena. The transmission peaks corresponding to the lower-band edges are hardly visible in the first passband for the case of fourand eight-layer structure, because of significant losses in the graphene sheets. Nevertheless, there are as many peaks as slabs in the second passband. Similar effects have been reported with the stack of metallic sub-wavelength meshes separated by identical dielectric slabs at microwaves, ${ }^{3}$ and the underlying physics has been explained in relation to band-gap properties of the corresponding infinite structure. The same explanation of bandpass and band-stop behavior is applicable for the case of a graphene-dielectric stack considered here at lowterahertz frequencies. It should also be noted that the bandpass and band-stop behavior is dependent on the geometrical and material parameters of the dielectric slabs and graphene sheets, but not on the overall length of the multilayer structure.

As an example, we consider the case of the four-layered graphene structure, with the same parameters of the dielectric layer used in the calculations of Fig. 3, but with different values of the chemical potential $\mu_{c}$ (electrostatic bias) for the graphene sheets. The transfer-matrix results for the transmissivity/reflectivity of the structure are depicted in Fig. 5, along with the FEM/HFSS results. It can be observed that there is no significant change in the frequency corresponding to the upper-band edge for $\mu_{c}=0.5$ and $1 \mathrm{eV}$. However, there is a considerable shift in the frequency corresponding to the lower-band edge. Also, it is noticed that the upper frequency band edge is the FP limit of the single dielectric layer (without graphene sheets), and the lower-band edge depends largely on the graphene impedance controlled by the chemical potential. This observation is consistent with the theory reported in Ref. 3 for mesh grid-dielectric stack at microwaves. Thus, by
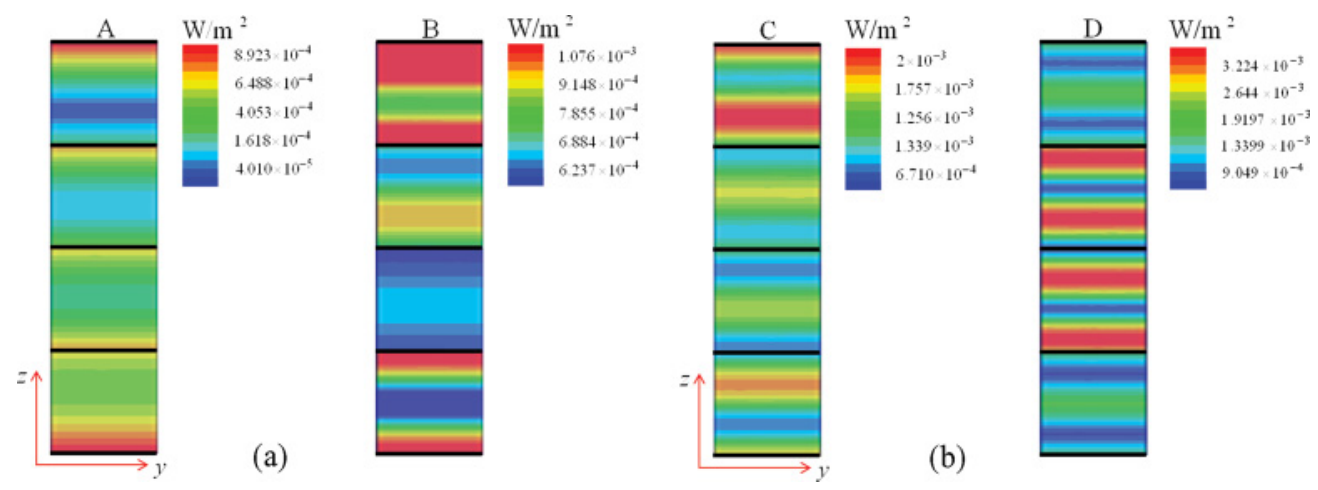

FIG. 8. (Color online) Reactive power distributions of the four resonance modes in the four-layer graphene-dielectric stack calculated using HFSS. 


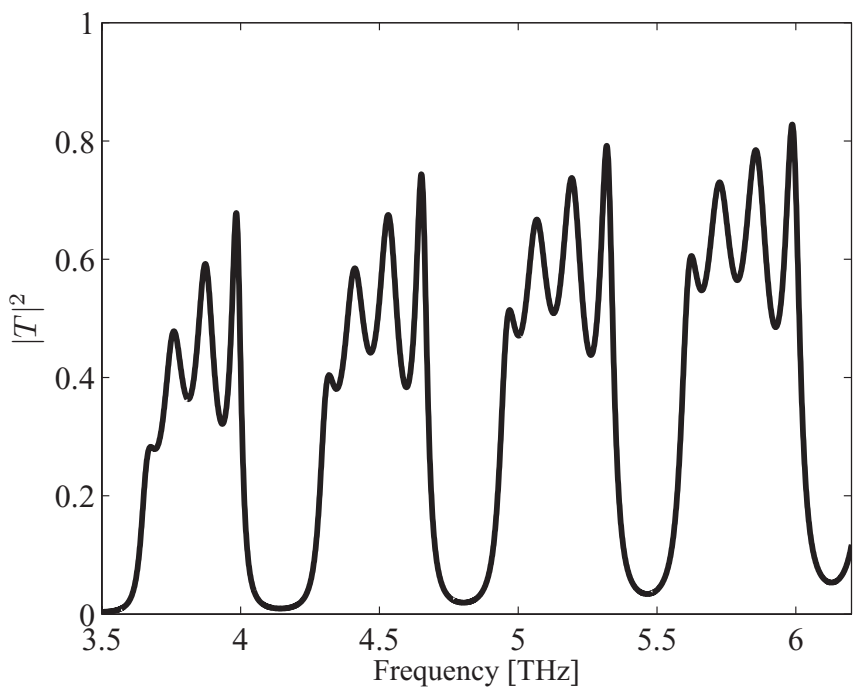

FIG. 9. Transfer-matrix results for the transmissivity $|T|^{2}$ of a four-layer graphene-dielectric stack. Structural parameters: $h=$ $150 \mu \mathrm{m}, \varepsilon_{r}=2.2$, and $\mu_{c}=1 \mathrm{eV}$.

varying the chemical potential of the graphene sheets (without changing the structural parameters), the transmission band (bandpass) of the structure can be controlled.

In Fig. 6, we plot the tangential electric-field distributions predicted by the transfer-matrix approach for the four transmission peaks that can be observed in Fig. 5 for the case of $\mu_{c}=1 \mathrm{eV}$ in the first transmissivity band (labeled in Fig. 6 as modes A, B, C, and D, calculated at the resonant frequencies of $1.843,2.353,3.099$, and $4.011 \mathrm{THz}$, respectively) along the propagation direction $z$. It should be noted that the the lower-band edge (mode A at $1.843 \mathrm{THz}$ ) is chosen at the frequency corresponding to the minimum of reflectivity [shown in Fig. 5(b)], and also it is observed that at this frequency the electric fields in the individual coupled graphene-dielectric cavities oscillate in phase with each other. ${ }^{3}$ It can be observed that each of the four resonance modes is associated with a specific field pattern along the propagation direction $(z)$. The field values are relatively small over each of those internal graphene sheets for mode D. For mode A, none of the internal graphene sheets have low electric-field values. The observed electric-field distributions for the resonance modes are qualitatively analogous to that observed in a mesh grid-dielectric stack at microwaves. ${ }^{3}$ It should be noted that the effect of an inductive reactance at the end of a transmission line section (as a dielectric slab loaded with graphene sheets) with a significant voltage excitation is related to an increase of the apparent (or equivalent) length of that section.
In addition, in Fig. 7 we present the magnitude of the total electric-field distributions in the four-layer graphene-dielectric stack calculated with HFSS (with the same parameters as in Fig. 5 for $\mu_{c}=1 \mathrm{eV}$ ). The results are obtained at the resonant frequencies of the modes $\mathrm{A}, \mathrm{B}, \mathrm{C}$, and $\mathrm{D}$, clearly demonstrating the field distributions associated with those shown in Fig. 6. Reactive power distributions in the same four-layer graphene-dielectric stack are calculated with HFSS at the resonant frequencies of the modes A, B, C, and D, as shown in Fig. 8. For mode A, it can be clearly seen that the reactive power is concentrated around the graphene sheets. For modes $\mathrm{B}$ and $\mathrm{D}$, the power level is low in the middle graphene sheet (due to null of the electric field), which is consistent with the electric-field distributions shown in Figs. 6 and 7.

A final example concerns the mechanical properties of graphene in the multilayered environment. In order to fabricate a graphene-dielectric stack, a thicker dielectric substrate is sometimes needed for mechanical handling of graphene. ${ }^{23}$ As one example of this case, in Fig. 9 the calculations based on the transfer-matrix approach for the transmission response of the four-layered graphene structure formed by thick dielectric slabs (with $h=150 \mu \mathrm{m}$ and $\varepsilon_{r}=2.2$ ) are shown. It can be observed that the structure exhibits a series of bandpass regions separated by the band gaps, similar to the previous examples.

\section{CONCLUSION}

Transmission properties of various graphene-dielectric stacks have been analyzed at low-terahertz frequencies. Similar transmission behavior through graphene-dielectric stack has been noticed with respect to the wire mesh grid-dielectric stack. The characteristics of the bandpass region (consisting of transmission peaks) of the transmission spectra are explained in terms of Fabry-Pérot resonances, similar to what was previously found for wire mesh grid-dielectric stacks at microwave frequencies. The study has been carried using the transfer-matrix approach, and an independent verification has been provided with the commercial numerical simulations. The considered structures can be useful in the design of tunable broadband planar filters.

\section{ACKNOWLEDGMENTS}

This work has been partially supported by the Spanish Ministry of Science and Innovation and European Union FEDER funds (projects TEC2010-16948 and CSD2008-00066) and by the Spanish Junta de Andalucia (Project TIC-4595). *yakovlev@olemiss.edu

${ }^{1}$ M. Scalora, M. J. Bloemer, A. S. Pethel, J. P. Dowling, C. M. Bowden, and A. S. Manka, J. Appl. Phys. 83, 2377 (1998).

${ }^{2}$ M. R. Gadsdon, J. Parsons, and J. R. Sambles, J. Opt. Soc. Am. B 26, 734 (2009).
${ }^{3}$ C. S. R. Kaipa, A. B. Yakovlev, F. Medina, F. Mesa, C. A. M. Butler, and A. P. Hibbins, Opt. Express 18, 13309 (2010).

${ }^{4}$ K. S. Novoselov, A. K. Geim, S. V. Morozov, D. Jiang, Y. Zhang, S. V. Dubonos, I. V. Grigorieva, and A. A. Firsov, Science 306, 666 (2004).

${ }^{5}$ A. K. Geim and K. S. Novoselov, Nat. Mater. 6, 183 (2007). 
${ }^{6}$ A. Reina, X. Jia, J, Ho, D. Nezich, H. Son, V. Bulovic, M. S. Dresselhaus, and J. Kong, Nano Lett. 9, 30 (2009).

${ }^{7}$ X. Li, W. Cai, J. An, S. Kim, J. Nah, D. Yang, R. Piner, A. Velamakanni, I. Jung, E. Tutuc, S. K. Banerjee, C. Colombo, and R. S. Ruoff, Science 324, 1312 (2009).

${ }^{8}$ A. Vakil and N. Engheta, Science 332, 1291 (2011).

${ }^{9}$ E. G. Mishchenko, A. V. Shytov, and P. G. Silvestrov, Phys. Rev. Lett. 104, 156806 (2010).

${ }^{10}$ G. W. Hanson, J. Appl. Phys. 104, 084314 (2008).

${ }^{11}$ V. V. Popov, T. Y. Bagaeva, T. Otsuji, and V. Ryzhii, Phys. Rev. B 81, 073404 (2010).

${ }^{12}$ M. Dragoman, D. Neculoiu, A. Cismaru, A. A. Muller, G. Deligeorgis, G. Konstantinidis, D. Dragoman, and R. Plana, Appl. Phys. Lett. 99, 033112 (2011).

${ }^{13}$ A. Yu. Nikitin, F. Guinea, F. J. Garcia-Vidal, and L. Martin-Moreno, Phys. Rev. B 84, 195446 (2011).
${ }^{14}$ G. W. Hanson, A. B. Yakovlev, and A. Mafi, J. Appl. Phys. 110, 114305 (2011).

${ }^{15}$ J. T. Kim and S.-Y. Choi, Opt. Express 19, 24557 (2011).

${ }^{16}$ S. A. Mikhailov, Europhys. Lett. 79, 27002 (2007).

${ }^{17}$ F. Rana, IEEE Trans. Nanotechnol. 7, 91 (2008).

${ }^{18}$ P.-Y. Chen and A. Alu, ACS Nano 5, 5855 (2011).

${ }^{19}$ G. W. Hanson, J. Appl. Phys. 103, 064302 (2008).

${ }^{20}$ V. P. Gusynin, S. G. Sharapov, and J. P. Carbotte, J. Phys.: Condens. Matter 19,026222 (2007).

${ }^{21}$ B. A. Munk, Frequency Selective Surfaces: Theory and Design (Wiley, New York, 2000).

${ }^{22}$ HFSS: High Frequency Structure Simulator based on the Finite Element Method, ver. 12.0, Ansoft Corporation [http://www. ansoft.com].

${ }^{23} \mathrm{Hao}$ Xin (private communication). 